\title{
Out-of-Band Interference in 5G mmW Multi-Antenna Transceivers: Co-existence Scenarios
}

\author{
Marko E. Leinonen, Nuutti Tervo, Olli Kursu, Aarno Pärssinen \\ University of Oulu, Oulu, Finland \\ email: \{marko.e.leinonen, nuutti.tervo, olli.kursu, aarno.parssinen\}@oulu.fi
}

\begin{abstract}
Large antenna arrays used for compensating the millimeter wave $(\mathrm{mmW})$ path loss gives interesting perspectives for considering out-of-band emissions and reception signals to be direction dependent. In addition, co-existence of lower frequency and $\mathrm{mmW}$ systems set special requirements for both conventional cellular and $\mathrm{mmW}$ systems. This paper presents discussion on these two cases as the most important out-of-band interference scenarios in fifth generation systems. Effects of intermodulation and adjacent channel leakage are discussed in both $\mathrm{mmW}$ beamforming transceiver and receiver, respectively. It is concluded that distortion is behaving differently in multi-antenna transmitter than at the receiver. Over-the-Air (OTA) measured and simulated examples of the nonlinear behavior of an array are given for receiver and transmitter, respectively. Furthermore, smart array linearization scheme given as a reference provides new perspective to consider the direction dependent out-of-band distortion as a part of the future cellular standards.
\end{abstract}

Keywords-ACLR, Arrays, Co-existence, Intermodulation, $3 G P P, 5 G$.

\section{INTRODUCTION}

One of the key drivers for the development of the next generation of communication systems, mostly referred as fifth generation $(5 \mathrm{G})$, is the envisioned ten-folded data rates compared with the current Long Term Evolution (LTE) (20 Gbps and 10 Gbps peak rates for downlink and uplink, respectively). One of the first $5 \mathrm{G}$ proof of concept $\mathrm{mmW}$ wireless backhaul system was showcased during the 2018 Winter Olympic Games in Korea and it was developed in 5GChampion project [1]. First third generation partnership project (3GPP) standards for $5 \mathrm{G}$ have been released at end of the 2017 and those include new radio (NR) interface requirements for both currently used frequencies and millimeter wave frequency bands [2]. The first commercial implementation of the $5 \mathrm{G} \mathrm{mmW}$ system will be based on non-standalone architecture, which leverage the LTE and NR air interfaces and current LTE core network. The first network deployments are expected early 2019 [3].

The large variety of operational frequency bands of cellular and other wireless systems, and combinations of those, will increase significantly with new 3GPP standard releases. Aggregated combinations of conventional sub- $6 \mathrm{GHz}$ and $\mathrm{mmW}$ bands are necessary for successful and reliable communications in different propagation environments and user scenarios. Hence, the co-existence requirement of the different frequency bands appear even within the same radio unit. The first $3 \mathrm{GPP}$ $5 \mathrm{G}$ NR standard [2] includes some requirements for the interoperability between RF bands. However, not all combinations have been identified and the discussion on the requirements is still ongoing. One of the unspecified interference scenarios is the co-existence of $5 \mathrm{G} \mathrm{mmW}$ and LTE LAA (Licensed Assisted Access) systems. Whereas the lower mmW bands are specified around $28 \mathrm{GHz}$, the operational band for LTE LAA is between 5 and $6 \mathrm{GHz}$. The $5 \mathrm{GHz}$ band $\mathrm{Wi}-\mathrm{Fi}$, based on IEEE 802.11 standards, shares the same operational frequency band with LTE LAA. The $5 \mathrm{GHz}$ Wi-Fi and LTE LAA radios may generate spurious harmonics to the $5 \mathrm{G} \mathrm{mmW}$ band causing interoperability problems. These harmonics may desensitize the $5 \mathrm{G} \mathrm{mmW}$ receiver. Moreover, many of the devices designed for sub- $6 \mathrm{GHz}$ frequencies have not been strictly specified and measured in the presence of co-located $\mathrm{mmW}$ systems. A large dynamic between path losses of currently used sub-6 GHz and $\mathrm{mmW}$ frequencies arises a scenario where the interfering lower frequency system might have significant transmission power compared with received $\mathrm{mmW}$ signal. A multi-radio interoperability between different wireless systems has not formally standardized in the 3GPP standard or by any other standardization body. Furthermore, it is not widely understood how $\mathrm{mmW}$ beamforming receiver and transmitter treats the out-of-band emissions and spurious responses in practice.

The $5 \mathrm{G}$ mmW radio signals face higher path loss than lower frequency LTE signals. Both transmission and reception devices will use MIMO (multiple input multiple output) antenna arrays to improve the radiated transmission and reception performance. The transmission (TX) and reception (RX) beams may be narrowed to improve the coverage and to reduce the radio interference from unwanted directions [4]. Moreover, adaptive beam forming will be used to direct radiated signals dynamically to the optimal direction. For example, a base station can dynamically follow the movement of the user. The radiated spectral mask of the antenna array is required to fulfill the 3GPP out-of-band emission specification. This requirement is either a conductive or a radiated requirement based on the base station type [2]. Conductive requirements are straightforward to characterize and measure. However, radiated out-of-band interference includes the effects of antenna array, thus the distortion becomes as direction dependent figure of merit. Especially, if parallel nonlinearities over the array have differences with each other, the beam pattern of the nonlinearity may differ from the beam pattern of the linear signal [5].

Radio performance requirements for previous generation for 3GPP standards GSM (Global System for Mobile Communications), WCDMA (Wideband Code Division Multiplexing Access) and LTE have been specified with conducted measurements, only. The 5G NR standard below 6 
$\mathrm{GHz}$ has conductive radio requirements but for $\mathrm{mmW}$ bands radio performance is specified with OTA, only [2]. Accurate OTA power measurements at $\mathrm{mmW}$ frequencies require new measurement methods compared to previous 3GPP standards. Thus, the accuracy of the OTA measurements at $\mathrm{mmW}$ frequencies is an important topic in standardization and in academia.

In this paper, we focus on discussing the two different interoperability scenarios, which are: (1) mmW system coexisting with an interfering Wi-Fi or LTE LAA system and (2) Two co-located $5 \mathrm{G} \mathrm{mmW}$ systems operating at adjacent channels with respect to each other. Several other co-existence and interference scenarios are present in $5 \mathrm{G}$ systems, but these two are considered to have significant meaning from the standardization perspective.

The rest of the paper is organized as follows. Interference scenarios are studied in Section II. First interference scenario is between $5 \mathrm{G} \mathrm{mmW}$ and $\mathrm{Wi}-\mathrm{Fi} / \mathrm{LTE}$ LAA is presented. Then outof-band interference of $5 \mathrm{G} \mathrm{mmW}$ transmitter is studied with simulations. The out-of-band interference of $5 \mathrm{G} \mathrm{mmW}$ receiver is studied and linearity measurement results of proof-of-concept receiver are shown. Finally, the conclusions based on the analysis are drawn.

\section{INTEROPERABILITY SCENARIOS}

Radio interoperability is a multi-dimensional optimization topic. There is no a single solution to guarantee that multiple radios operating at different frequency bands can co-exist without interference. The radio interoperability can be defined, for example, with a spectrum consumption model, which is described in IEEE standard 1900.5.2 [6]. The spectrum model includes following parameters that define radio interoperability:

- $\quad$ Conducted transmission power

- $\quad$ Spectrum mask of transmission and reception signals

- $\quad$ Antenna directivity

- $\quad$ Propagation loss model

- $\quad$ Reception intermodulation signal mask

- Transmission and reception timings

- Location and transmission starting time

The spectrum model above is a generic model for the radio interoperability where all aspects of the radio transmission and

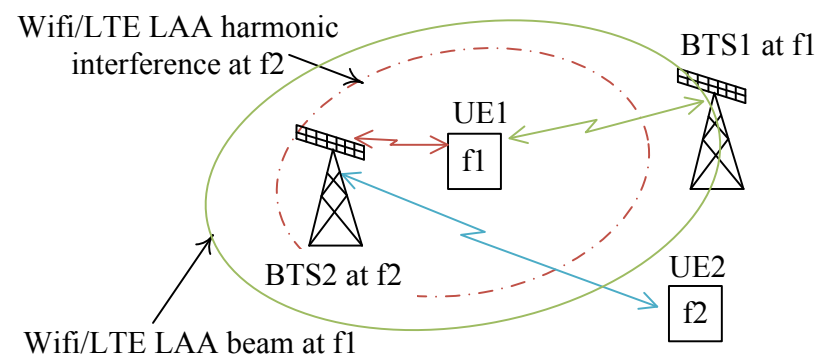

Fig. 1. Wi-Fi/LTE LAA harmonic interference scenario to $5 \mathrm{G} \mathrm{mmW}$.

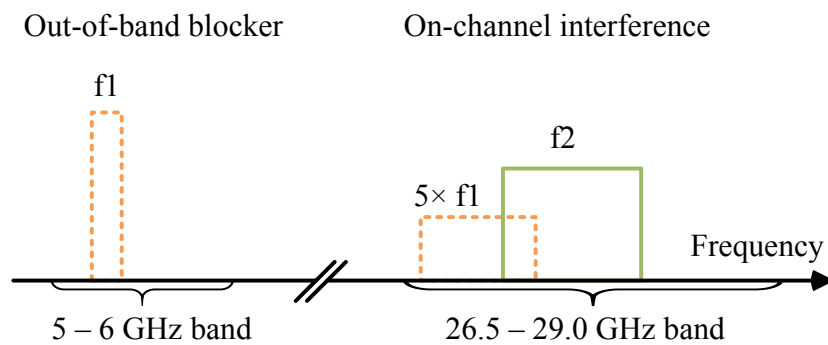

Fig. 2. Wi-Fi/LTE LAA band interference scenarios to $5 \mathrm{G} \mathrm{mmW}$ band.

reception are taken into account. In our studied interference scenarios, the interfering and the victim radios are near vicinity of each other.

\section{A. $5 G \mathrm{mmW}$ and LTE LAA / Wi-Fi interference}

It is assumed here that the $5 \mathrm{G} \mathrm{mmW,} \mathrm{Wi-Fi} \mathrm{and} \mathrm{LTE} \mathrm{LAA}$ systems will be deployed at the same indoor locations and these networks will overlay each other. This is a viable scenario as all these three systems may be implemented into one base station unit in order to provide full flexibility for the operators for network deployment. In some deployment scenarios, all these three systems need to operate simultaneously. An illustration of the network deployment scenario is shown in Fig. 1. The 5G $\mathrm{mmW}$ base station (BTS2) and user equipment 2 (UE) operate at $\mathrm{mmW}$ frequency of $\mathrm{f} 2$ and Wi-Fi/LTE LAA UE1 and BTS1 operate at frequency of $\mathrm{fl}$. The UE1 generates harmonic interference at $\mathrm{f} 2$ frequency overlapping the wanted signal and desensitizing the $5 \mathrm{G} \mathrm{mmW}$ receiver, as illustrated in Fig. 2. This scenario happens when Wi-Fi or LTE LAA terminal operates at close proximity of $5 \mathrm{G} \mathrm{mmW}$ BTS. For example, the $5 \mathrm{G} \mathrm{mmW}$ BTS is installed to ceiling of the corridor and user is uploading content via $\mathrm{Wi}-\mathrm{Fi} / \mathrm{LTE}$ LAA network located in close proximity of the $5 \mathrm{G}$ BTS.

The Wi-Fi/LTE LAA transmission itself is an out-of-band blocker for $5 \mathrm{G} \mathrm{mmW}$ receiver and it may be at high level in the $\mathrm{mmW}$ antenna and receiver due to smaller path loss than $\mathrm{mmW}$ signal. Linearity of the $5 \mathrm{G} \mathrm{mmW}$ receiver needs to tolerate, not only mmW band interference, but Wi-Fi/LTE LAA out-of-band blocker, as well. Maximum effective radiated isotropic power (EIRP) of Wi-Fi UE at $5 \mathrm{GHz}$ band is in USA $+24 \mathrm{dBm}$ and in Europe $+20 \mathrm{dBm}$ and the maximum conducted LTE LAA UE power is $+23 \mathrm{dBm}$. The Wi-Fi/LAA blocker level may be close to 3GPP LTE out-of-band requirement $-15 \mathrm{dBm}$ [2] in real life installation since at least 40 centimeter the guard distance is needed between radios. The $5 \mathrm{G} \mathrm{mmW}$ receiver can be implemented without pre-selection antenna filter and thus only filtering is coming from the frequency response of the antenna before non-linear amplifiers. RF architecture without dedicated RF band selection filter is used in [1] and expected to common approach to avoid lossy and large filtering elements at $\mathrm{mmW}$ frequencies.

The fifth harmonic of the LTE LAA or Wi-Fi transmission will generate harmonic interference falling to $28 \mathrm{GHz}$ band as shown in Fig. 2 . The $5^{\text {th }}$ harmonic interference of the $\mathrm{Wi}-\mathrm{Fi} / \mathrm{LTE}$ LAA transmission is five times wider than the communication transmission. For example, if LTE signal is $20 \mathrm{MHz}$ wide, the $5^{\text {th }}$ harmonic is $100 \mathrm{MHz}$ which is in the same range to the $5 \mathrm{G}$ 


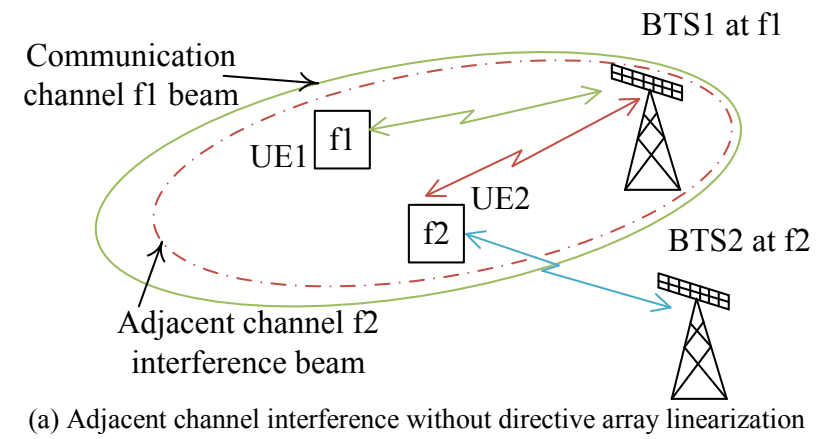

(a) Adjacent channel interference without directive array linearization

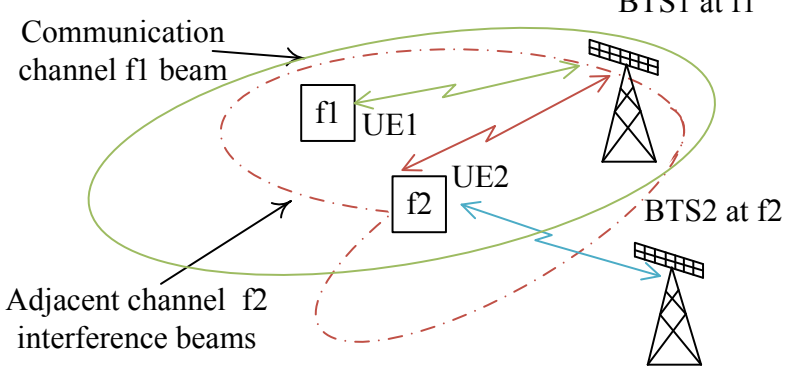

(b) Adjacent channel interference in $5 \mathrm{G} \mathrm{mmW}$ system with array linearization

Fig. 3. Adjacent channel TX interference scenarios of $5 \mathrm{G}$ mmW array.

mmW signal bandwidth. The Wi-Fi transmission may be up to $160 \mathrm{MHz}$ wide and the harmonic spurious is up to $800 \mathrm{MHz}$, which is wider than currently standardized $5 \mathrm{G} \mathrm{mmW}$ signal bandwidth of $400 \mathrm{MHz}$ [2]. Current 3GPP LTE LAA standard for band 46 for UE specifies that the interference transmission up to $26 \mathrm{GHz}$ needs to be below-30dBm @ $1 \mathrm{MHz}$ or $-17 \mathrm{dBm}$ @ $20 \mathrm{MHz}$ [7]. This requirement is not covering the whole 5G $\mathrm{mmW}$ frequency band and the specified harmonic power is significantly higher than not yet specified reference sensitivity level of $5 \mathrm{G} \mathrm{NR} \mathrm{mmW}$ receiver. Actual power levels of harmonics transmissions is mainly caused by the power amplifier (PA) and the operation class of it. The class A power amplifier produces lower harmonics as class $\mathrm{AB}$ or class $\mathrm{C}$ devices. The drawback of more linear operation of PA is the current consumption. For example, the proof-of-concept $5 \mathrm{G}$ $\mathrm{mmW}$ receiver has receiver sensitivity $-56 \mathrm{dBm}$ without antenna gains [1].

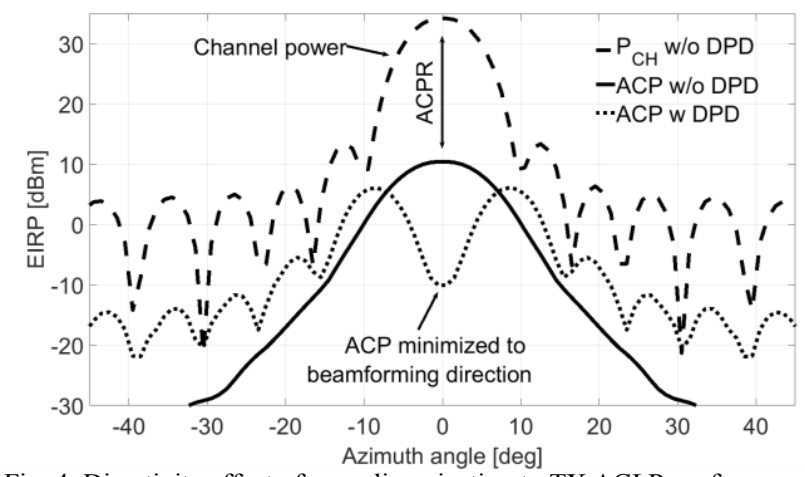

Fig. 4. Directivity effect of array linearization to TX ACLR performance.

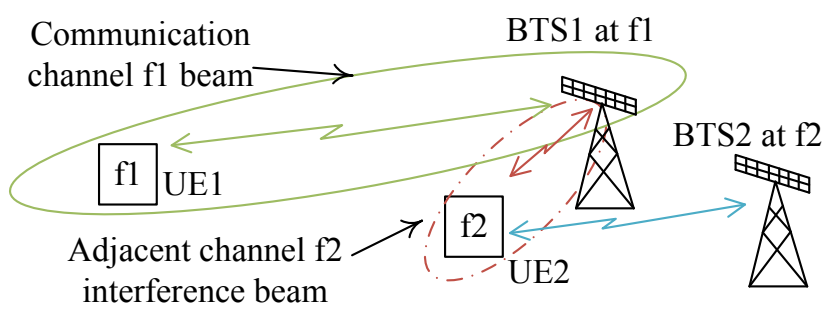

Fig. 5. Adjacent channel interference in receiver due to other $5 \mathrm{G} \mathrm{mmW}$ band transmission.

The multi-radio interoperability can be done with physical separation. A needed guard distance between interfering and the victim radios depends on the interfering signal power level, the sensitivity of the receiver, antenna gains and the allowed sensitivity reduction of the receiver. This interference scenario may occur between radios within in the same multi-radio unit operating simultaneously. A sufficient antenna isolation between transmission and reception antennas is needed to ensure multi-radio interoperability.

\section{B. $5 G \mathrm{~mm} W$ transmitter generated interference}

The transmission spectrum mask of the 3GPP 5G NR transmission is defined for the $\mathrm{mmW}$ base station transmitter with OTA measurement [2]. According to the standard, the TX ACLR (Adjacent Channel Leakage Ratio) requirement is defined as TRP (Total Radiated Power), where the transmission power is integrated over the whole space. The TX ACLR requirement for $5 \mathrm{G} \mathrm{mmW}$ bands is shown in Table I. These requirements are reduced from the LTE band requirement of -45 $\mathrm{dB}$ due to higher frequency and more demanding radio implementation. The ACLR is measured with OTA and the requirement is valid for the whole array.

Two adjacent channel transmission interference scenarios are illustrated in Fig. 3a and 3b. The Fig. 3a illustrates a case where the adjacent transmission channel power covers similar shape area than communication signal transmission. This happens when a single antenna (e.g. LTE system) or mmW antenna array without array linearization is used. The Fig. 3b illustrates a case where the adjacent transmission channel power has a different coverage than the communication signal. This case occurs with $\mathrm{mmW}$ antenna array with array linearization [5].

When discussing about array ACLR, we should keep in mind that an antenna array fundamentally only directs the power. Hence, the TRP ACLR (or absolute ACP (Adjacent Channel Power) is independent on the intended transmission direction. However, as it has been shown and discussed in [5], [8]-[12], the ACP may, in some cases, experience the array response differently than the fundamental signal. Such behavior can appear when multiple parallel nonlinear array elements (e.g. multiple PAs each driving one antenna element) has different shapes of nonlinearity or they are driven with different

TABLE I. OTA ACLR REQUIREMENT FOR 5G MMW RADIO

\begin{tabular}{|c|c|c|}
\hline Frequency band & $\mathbf{2 4 . 2 4}-\mathbf{3 3 . 4} \mathbf{~ G H z}$ & $\mathbf{3 7}-\mathbf{5 2 . 6} \mathbf{~ G H z}$ \\
\hline $\begin{array}{c}\text { ACLR TX } \\
\text { requirement }\end{array}$ & $-28.0 \mathrm{~dB}$ & $-26.0 \mathrm{~dB}$ \\
\hline
\end{tabular}




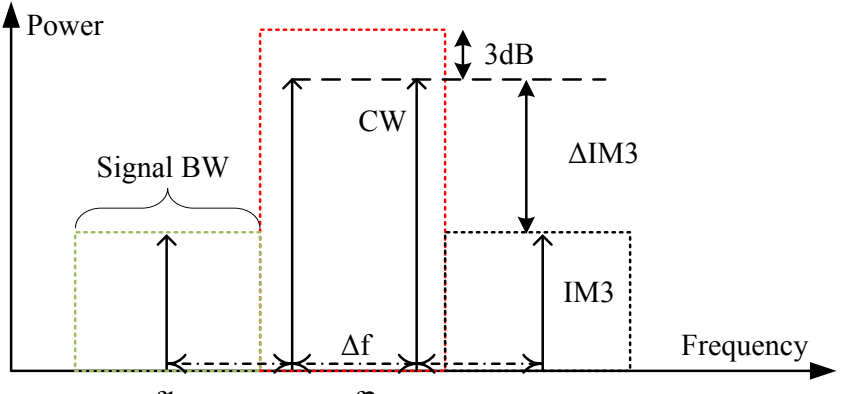

f1

f2

Fig. 6. Third order IMD measurement with $\mathrm{CW}$ and modulated signal.

amplitude levels. With directive array linearization schemes presented e.g. in [5], the differences can be even used to shape the beam of the ACP with respect to the fundamental signal. Similar effect of ACP spreading may happen also when multiple independent data signals (i.e. multiple streams) are transmitted from the same array such as shown in [5],[10]-[12]. However, without the differences between the nonlinearities of the parallel antenna branches, this effect does not happen in single-stream transmission.

An example of directive ACP is presented in Fig. 4. The figure demonstrates the channel and adjacent channel powers over the azimuth angle with and without a directive digital predistortion (DPD) scheme. The details of the linearization concept and the analysis flow resulting the directed ACP is presented in [5]. In this example, the ACLR observed in beamforming direction is with and without DPD is $-44 \mathrm{~dB}$ and $-23 \mathrm{~dB}$. However, corresponding ACLR values characterized as TRP are $-25 \mathrm{~dB}$ and $-22 \mathrm{~dB}$, with and without DPD, respectively. Hence, TX ACLR is directive, if differences over the parallel nonlinearities are utilized for linearization. TRP ACP specification does not necessarily guarantee the interference level between the two systems if the directivity is not taken into account in specifications.

\section{Adjacent channel interference to $5 G \mathrm{mmW}$ receiver}

The $\mathrm{mmW}$ receiver will face similar near-far problems than any other cellular receiver and this scenario is illustrated in Fig.

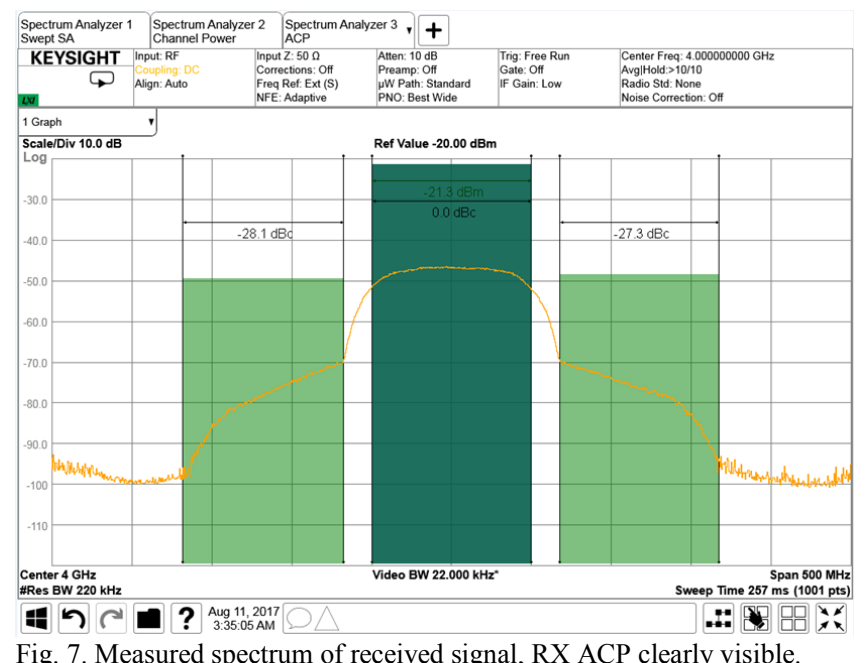

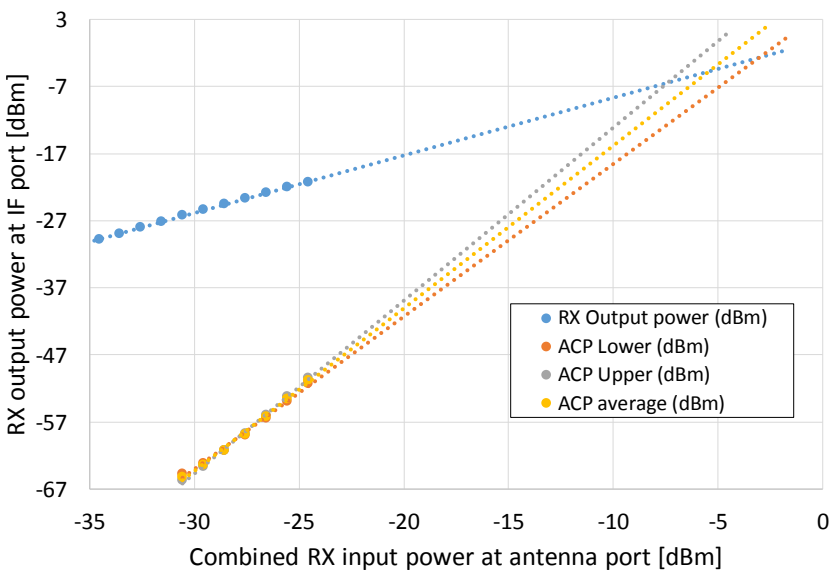

Fig. 8. Linearity measurement of array receiver to main beam direction.

5. The victim mmW receiver e.g. BTS1 operates at frequency $\mathrm{fl}$ and it communicates to UE1, which is at the edge of the cell. A UE2 connected to the BTS2 operates at frequency $\mathrm{f} 2$ nearby of the BTS1. When BTS1 receivers a weak signal at frequency $\mathrm{fl}$, simultaneously it receives a strong interference at the adjacent radio channel $\mathrm{f} 2$. The spectrum of the strong interference signal at $\mathrm{f} 2$ may spectrally grow over to the frequency f1 due to nonlinearities of the receiver. Thus, the spectral regrowth deteriorates the quality of the received signal.

The linearity of the receiver determines how strong interference the receiver can tolerate. When multiple signals interact in a non-linear component, a mixing process will generate new frequency components from the received signals. One of the most commonly used a merit of linearity of the receiver is the third-order intercept point (IP3) [13]. The thirdorder linearity can be measured with a two-tone test, which is shown in Fig. 6. Two CW (Continuous Wave) signals are fed into the receiver and mixing products $\Delta \mathrm{f}$ frequency apart from the test signals is measured. This two-tone measurement method is used for linearity measurement of array receiver in [14]. The input IP3 point (IIP3) can be calculated [13]

$$
I I P 3=\frac{1}{2(\Delta I M 3)}+P_{\text {tone }}
$$

where $P_{\text {tone }}$ is the power of one $\mathrm{CW}$ test signal and $\triangle \mathrm{IM} 3$ is a power difference between mixing product and one test tone signal. The CW signal is not optimal test signal for a wide band receiver and a modulated test signal is preferred.

We performed IP3 testing with a digitally modulated $100 \mathrm{MHz}$ test signal. The modulated test signal can be considered to be the same than two CW test signals in the two-tone test. The power of modulated test signal may be considered to be shared to both $\mathrm{CW}$ signals and (1) is modified for modulated signal case, so that $P_{\text {tone }}=P_{\text {signal_Bw }}-3 \mathrm{~dB}$.

We measured the linearity of the proof of concept $5 \mathrm{G} \mathrm{mmW}$ receiver with a $100 \mathrm{MHz}$ wide 16-QAM modulated test signal with a roll-off factor $\alpha=0.35$. The test signal was transmitted OTA to the antenna array of the $5 \mathrm{G} \mathrm{mmW}$ receiver at $26.5 \mathrm{GHz}$ frequency [1]. The receiver signal paths of $5 \mathrm{G} \mathrm{mmW}$ array receiver were phased so that the main lobe of the antenna array pointed towards the test antenna. The phasing of the receiver 
paths was done with digitally controlled phase shifters and each receiver path had own phase shifter. A spectrum at the output of the receiver was measured and one of the measurement result is shown in Fig. 7. This measurement was done with a high input power level in order to show clearly the RX ACP behavior of the received signal. The channel and ACP powers were measured by integrating the signal over the modulation bandwidth and avoiding the roll-off region of the signal. Similar ACP measurement method is used with WCDMA and LTE transmitter already. The measured spectrum shows clearly that the receiver has limited linearity since the received signal has significant spectral regrowth to the adjacent channel lower operating channel $\mathrm{fl}$ as illustrated in Fig. 6. The ACP value of the received signal in Fig 7. corresponds the IM3 level in the two-tone test in Fig. 6.

The level of the test signal was varied and a summary figure of the linearity measurement results is shown in Fig. 8. It can be seen that the output spectrum of the receiver is not completely symmetrical and the average of the lower and the higher side ACP results gave the most stable result. The measured IIP3 of the array receiver at $26.5 \mathrm{GHz}$ operational frequency is -5.4 $\mathrm{dBm}$, which can be seen from Fig. 8. The linearity of the first LNA dominates the linearity of the array receiver and the specification of the IIP3 of used LNA component is $-4.0 \mathrm{dBm}$. The third-order non-linearity expects that the slope of the $3^{\text {rd }}$ order intermodulation (IM) product is $3: 1$. Our measurement results show that the slope of the IM3 is 2.4 , which is a good match with the theoretical value.

The linearity of the $5 \mathrm{G} \mathrm{mmW}$ proof-of-concept receiver need to be validated at frequencies outside of $\mathrm{mmW}$ frequencies and future work includes the linearity measurements of the array receiver with Wi-Fi and LTE LAA test signals.

\section{CONCLUSIONS}

First version of the 3GPP standard for $5 \mathrm{G}$ NR mmW radios was released at end of 2017. The requirement of TX ACLR for $5 \mathrm{G} \mathrm{NR} \mathrm{mmW}$ transmission is specified with OTA measurement, only. The OTA ACLR, as a TRP, does not take into account the direction of the interference. We have introduced directive ACP in earlier papers. We emphasis that directive ACP would enable enhancement in environment where there are restricted geographical interference limitations like radars or zone edges.

Multi-radio interoperability requirements between 5G NR $\mathrm{mmW}$ radio and other radio systems like $\mathrm{Wi}-\mathrm{Fi}$ and LTE is standardized. We indicated that current LTE LAA standard do not cover $5 \mathrm{G} \mathrm{mmW}$ interoperability.

We evaluated the linearity of proof-of-concept $5 \mathrm{G} \mathrm{mmW}$ receiver with OTA measurement with a modulated $100 \mathrm{MHz}$ wide $\mathrm{mmW}$ test signal. The used linearity measurement method of the receiver was similar to the ACP testing of LTE transmitter. The measured linearity of the array receiver to the main beam direction follows a third order linearity assumption as expected.

\section{ACKNOWLEDGMENT}

The research leading to these results has received funding from the European Union H2020 5GPPP under grant n. 723247 and supported by the Institute for Information \& communications Technology Promotion (IITP) grant funded by the Korea government (MSIP) (No.B0115-16-0001, 5G CHAMPION). The authors are very grateful to Mr. Juho Rivinoja who performed linearity measurements of $5 \mathrm{G} \mathrm{mmW}$ receiver for the study.

\section{REFERENCES}

[1] M. E. Leinonen, G. Destino, O. Kursu, M. Sonkki and A. Pärssinen, "28 $\mathrm{GHz}$ Wireless Backhaul Transceiver Characterization and Radio Link Budget," ETRI J., vol. 40, no. 1, Feb. 2018, pp. 89-100

[2] Base Station (BS) radio transmission and reception (Release 15) 3GPP TS 38.104 V15.0.0, Dec. 2017

[3] L. Casaccia, "Live from Lisbon: 3GPP completes first 5G standard," OnQ Blog, Dec. 20, 2017. Accessed: Mar. 1, 2018.

[4] F. Boccardi, R. W. Heath, A. Lozano, T. L. Marzetta, P. Popovski, "Five Disruptive Technology Directions for 5G," IEEE Commun. Mag., vol. 52, no. 2, Feb. 2014, pp. 74-80.

[5] N. Tervo, J. Aikio, T. Tuovinen, T. Rahkonen and A. Parssinen, "Digital predistortion of amplitude varying phased array utilising over-the-air combining," 2017 IEEE MTT-S International Microwave Symposium (IMS), Honololu, HI, 2017, pp. 1165-1168.

[6] J. A. Stine, "The IEEE 1900.5.2 Standard for Spectrum Consumption Modelling", Mitre Corporation, 2014

[7] User Equipment (UE) radio transmission and reception (Release 15) 3GPP TS 36.101 V15.2.0, Mar. 2018

[8] S. Kim, E. Visotsky, P. Moorut, K. Bechta, A. Ghosh and C. Dietrich, "Coexistence of $5 \mathrm{G}$ With the Incumbents in the 28 and $70 \mathrm{GHz}$ Bands," in IEEE Journal on Selected Areas in Communications, vol. 35, no. 6, pp. 1254-1268, June 2017.

[9] K. Hausmair et al., "Prediction of Nonlinear Distortion in Wideband Active Antenna Arrays," in IEEE Transactions on Microwave Theory and Techniques, vol. 65, no. 11, pp. 4550-4563, Nov. 2017.

[10] E. G. Larsson and L. Van der Perre, "Out-of-Band Radiation from Antenna Arrays Clarified," in IEEE Wireless Communications Letters, vol. PP, no. 99, pp. 1-1.

[11] C. Moll' en, U. Gustavsson, T. Eriksson, and E. G. Larsson, "Out-of-band radiation measure for MIMO arrays with beamfored transmission," in Proc. IEEE Int. Conf. on Commun. (ICC), 2016

[12] N. Tervo, J. Aikio, T. Tuovinen, T. Rahkonen and A. Parssinen, "Effects of PA Nonlinearity and Dynamic Range in Spatially Multiplexed Precoded MIMO Systems," European Wireless 2016; 22th European Wireless Conference, Oulu, Finland, 2016, pp. 1-6.

[13] J. H. Reed, Software Radio: A Modern Approach to Radio Engineering. Upper Saddle River, NY, USA: Prentice Hall PTR, 2002.

[14] R. G. Gatti, M. Dionigi and R. Sorrentino, "Computation of Gain, Noise Figure, and Third-Order Intercept Point of Active Array Antennas," IEEE Trans. Antennas Propag., Vol. 52, No. 11, Nov. 2004, pp. 3139-3142 